\title{
P073: Outbreak of carbapenemase-producing Pseudomonas aeruginosa in a tertiary care hospital
}

\author{
P Navarro, M Cantero Caballero, I Wijers, I Cerón, E Martinez de Albeniz, P Rodriguez \\ From 2nd International Conference on Prevention and Infection Control (ICPIC 2013) \\ Geneva, Switzerland. 25-28 June 2013
}

\section{Introduction}

Carbapenemase-mediated resistance to carbapenems in Pseudomonas Aeruginosa has increased in Spain since 2003.

\section{Objectives}

To describe an outbreak of multi-resistant carbapenemase-producing Pseudomonas Aeruginosa, in a bone marrow transplant unit (BMTU) of a tertiary care hospital.

\section{Methods}

Descriptive study of the outbreak and the control measures implemented. Surveillance cultures from patients staying in the BMTU were taken in order to detect colonization, as well as environmental samples.

\section{Results}

In March 2012 a carbapenemase-producing P. Aeruginosa isolate resistant to carbapenems and beta-lactam antibiotics was isolated from a wound culture of a patient admitted to our BMTU. The patient had previously presented a sepsis secondary to ecthyma gangrenosum caused by carbapenem-resistant but non-carbapenemaseproducing $P$. Aeruginosa and had prolonged broadspectrum antibiotic therapy. Carbapenemase-producing P. Aeruginosa was isolated from all of the following cultures. During the month of April 2012 two new cases were identified on the BMTU, both suffering from symptomatic urinary tract infections, with detection of P. Aeruginosa in urine cultures. PCR was used to confirm that it was the same VIM-type strain in all of the three cases. Control measures included: contact isolation in individual rooms, specialized personnel attending the isolated patients, enhanced standard precautions, additional cleaning of patient rooms and enhanced cleaning and disinfection of medical materials. In June 2012, two of the three patients were discharged and one of them died from an unrelated cause. No new cases have been detected on the BMTU.

\section{Conclusion}

The capacity of microorganisms, and especially $P$. Aeruginosa, to acquire new mechanisms of resistance under antibiotic selection pressure, poses important therapeutic problems and difficulties for the control of healthcareassociated infections.

\section{Disclosure of interest}

None declared.

Published: 20 June 2013

doi:10.1186/2047-2994-2-S1-P73

Cite this article as: Navarro et al:: P073: Outbreak of carbapenemaseproducing Pseudomonas aeruginosa in a tertiary care hospital. Antimicrobial Resistance and Infection Control 2013 2(Suppl 1):P73. 